Pesq. Vet. Bras. 37(3):241-247, março 2017 DOI: $10.1590 / \mathrm{S} 0100-736 \mathrm{X} 2017000300007$

\title{
Uso de baixa dose de ACTH sintético no teste de estimulação da função adrenal para o diagnóstico e controle do hiperadrenocorticismo canino: avaliação da eficácia diagnóstica1
}

\author{
Renata C.B. Martins ${ }^{2 *}$ e Márcia M. Jericó ${ }^{3}$
}

\begin{abstract}
Martins R.C.B. \& Jericó M.M. 2017. [Use of low-dose of synthetic ACTH in the adrenal stimulation test for diagnosis and monitoring of canine hyperadrenocorticism: evaluation of diagnostic efficacy.] Uso de baixa dose de ACTH sintético no teste de estimulação da função adrenal para o diagnóstico e controle do hiperadrenocorticismo canino: avaliação da eficácia diagnóstica. Pesquisa Veterinária Brasileira 37(3):241-247. Curso de Medicina Veterinária, Universidade Anhembi Morumbi (UAM), Rua Doutor Almeida Lima 1134, Parque da Mooca, São Paulo, SP 03164-000, Brazil. E-mail: renatacast.vet@gmail.com

ACTH stimulation is the gold standard test to monitor levels of endogen control of patients under treatment for canine hyperadrenocorticism and it may also be used to diagnose the disease. Current protocols use doses ranging from $5 \mathrm{ug} / \mathrm{kg}$ to $250 \mathrm{ug}$ per animal by intravenous or intramuscular administration. There are no studies with doses inferior to $5 \mathrm{ug} / \mathrm{kg}$ in adrenopatic patients. In the present investigation, the dose of $1 \mathrm{ug} / \mathrm{kg} / \mathrm{IV}$ was tested; compared with the recommended dose of $\mathrm{d} 5 \mathrm{ug} / \mathrm{kg} / \mathrm{IV}$ in groups of dogs under clinical suspicion of HAC (HAC Diag), animals under treatment for HAC (HAC Control) and healthy animals (Healthy). Under the dose of $1 \mathrm{ug} / \mathrm{kg} / \mathrm{IV}$, average results for baseline cortisol values were equal to $2.40 \mathrm{ug} / \mathrm{dL}(+/-1.57 \mathrm{ug} / \mathrm{dL})$ for healthy, $1.53 \mathrm{~g} / \mathrm{dL}(+/-0.93 \mathrm{ug} / \mathrm{dL})$ for $\mathrm{HAC}$ Control and 3.37ug/dL(+/-1.57ug/dL) for HAC Diag. Post-ACTH values in the dose of $1 \mathrm{ug} /$ $\mathrm{kg}$ were average $11.43 \mathrm{ug} / \mathrm{dL}(+/-2.46 \mathrm{ug} / \mathrm{dL})$ for healthy animals, $2.67 \mathrm{ug} / \mathrm{dL}(+/-1.39 \mathrm{ug} /$ $\mathrm{dL})$ for HAC Control and $16.56 \mathrm{ug} / \mathrm{dL}(+/-7.62 \mathrm{ug} / \mathrm{dL})$ for HAC Diag group. Basal cortisol values at a dose of $5 \mathrm{ug} / \mathrm{kg}$ were $0.89 \mathrm{ug} / \mathrm{dL}(+/-0.23 \mathrm{ug} / \mathrm{dL})$ Control group for HAC; average $3.08 \mathrm{ug} / \mathrm{dL}(+/-1.99 \mathrm{ug} / \mathrm{dL})$ for HAC Diag group. Baseline cortisol under the dose of $5 \mathrm{ug} /$ $\mathrm{kg}$ were average $3.71 \mathrm{ug} / \mathrm{dL}(+/-1.57 \mathrm{ug} / \mathrm{dL})$ for HAC Control and $22.52 \mathrm{~g} / \mathrm{dL}(+/-8.75 \mathrm{ug} / \mathrm{dL})$ for HAC diag. Based on the present results, it was found that both doses of 1 and $5 \mathrm{ug} / \mathrm{kg}$ of synthetic ACTH do not differ, providing the same kind of change in cortisol values (ANOVA, $\mathrm{p}=0.225$ ). Also, the dose of $1 \mathrm{ug} / \mathrm{kg}$ of ACTH was equally effective in raising levels of cortisol in the three groups tested (Healthy, HAC and HAC Control Diag; ANOVA, $\mathrm{p}<0.05$ ). Through the Dunn test it was observed that HAC Control presented HAC- $\Delta$ cortisol (delta = difference between cortisol after stimulation and basal cortisol) significantly lower than HAC Diag $(p<0.05)$ and healthy animals $(p<0.05)$. Therefore the dose of $1 \mathrm{ug} / \mathrm{kg}$ of synthetic ACTH can be effectively used to perform the ACTH stimulation test effectively.
\end{abstract}

INDEX TERMS: ACTH, adrenal stimulation test, hyperadrenocorticism, hypercortisolism, cortisol, dogs, endocrine diseases, cortrosin.

\footnotetext{
${ }^{1}$ Recebido em 31 de março de 2015.

Aceito para publicação em 16 de Junho de 2016.

${ }^{2}$ Discente de iniciação científica do Curso de Medicina Veterinária, Universidade Anhembi Morumbi (UAM), Rua Doutor Almeida Lima 1134, Parque da Mooca, São Paulo, SP 03164-000, Brasil. *Autor para correspondência: renatacast.vet@gmail.com

${ }^{3}$ Docente do Departamento de Clínica Médica do Hospital Veterinário Anhembi Morumbi, UAM, Rua Doutor Almeida Lima 1134, Parque da Mooca, São Paulo, SP 03164-000, Brasil.
}

RESUMO.- 0 teste de estimulação com ACTH é o teste de escolha para realizar o controle dos valores de cortisol endógeno em pacientes sob tratamento para o hiperadrenocorticismo canino, podendo ser utilizado também para diagnosticar a doença. Os protocolos atuais utilizam doses que variam entre $5 \mathrm{ug} / \mathrm{kg}$ a $250 \mathrm{ug} /$ animal por administração intravenosa ou intramuscular. Não se constatam estudos com doses menores que as de $5 \mathrm{ug} / \mathrm{kg}$ em pacientes portadores 
de hiperadrenocorticismo. No presente estudo, foi testada a dose de $1 \mathrm{ug} / \mathrm{kg} / \mathrm{IV}$; comparada à dose consagrada de $5 \mathrm{ug} /$ $\mathrm{kg} / \mathrm{IV}$, em grupos de animais suspeitos de HAC (HAC Diag), animais portadores de HAC e em tratamento (HAC Control) e animais sadios (Sadios). Na dose de $1 \mathrm{ug} / \mathrm{kg} / \mathrm{IV}$, os valores basais de cortisol dos Sadios foram iguais à média 2,40ug/ $\mathrm{dL}(+/-1,57 \mathrm{ug} / \mathrm{dL})$, dos HAC control foi de média 1,53ug/ $\mathrm{dL}(+/-0,93$,ug/dL) e dos HAC diag foi média 3,37ug/dL(+/$1,57 \mathrm{ug} / \mathrm{dL}$ ). Os valores pós-ACTH na dose de $1 \mathrm{ug} / \mathrm{kg}$ foram de média 11,43ug/dL(+/-2,46ug/dL) para animais sadios, $2,67 \mathrm{ug} / \mathrm{dL}(+/-1,39 \mathrm{ug} / \mathrm{dL})$ para o grupo HAC Control e média 16,56ug/dL(+/-7,62ug/dL) para o grupo HAC Diag. Os valores basais de cortisol na dose de $5 \mathrm{ug} / \mathrm{kg}$ foram $0,89 \mathrm{ug} /$ dL(+/-0,23ug/dL) para o grupo HAC Control; média 3,08ug/ $\mathrm{dL}(+/-1,99 \mathrm{ug} / \mathrm{dL})$ para o grupo HAC Diag. Os valores pósACTH na dose de $5 \mathrm{ug} / \mathrm{kg}$ foram de média $3,71 \mathrm{ug} / \mathrm{dL}(+/-$ $1,57 \mathrm{ug} / \mathrm{dL}$ ), para o grupo HAC control e média $22,52 \mathrm{ug} / \mathrm{dL}$ (+/-8,75ug/dL) para o grupo HAC diag. Analisando-se os resultados obtidos, constatou-se que as doses 1 e $5 \mathrm{ug} / \mathrm{kg}$ de ACTH sintético não diferem entre si, promovendo o mesmo tipo de variação nos valores de cortisol (ANOVA; $p=0,225$ ). Também, que a dose de $1 \mathrm{ug} / \mathrm{kg}$ de ACTH foi igualmente eficaz na elevação dos níveis de cortisol nos três grupo testados (Sadios, HAC Control e HAC Diag; ANOVA, $p<0,05$ ). E, pelo teste de Dunn observamos que o grupo HAC control apresenta $\Delta$-cortisol (delta =diferença entre cortisol após estimulo e o cortisol basal) significativamente menor que o dos grupos diagnóstico $(\mathrm{p}<0,05)$ e animais sadios $(\mathrm{p}<0,05)$. Concluiu-se que a dose de $1 \mathrm{ug} / \mathrm{kg}$ de ACTH sintético pode ser utilizada com eficácia para a realização do teste de estimulação com ACTH.

TERMOS DE INDEXAÇÃO: ACTH, teste de estimulação da função adrenal, hiperadrenocorticismo canino, hipercortisolismo, cortisol, cães, endocrinopatias, cortrosina.

\section{INTRODUÇÃO}

O (HAC) canino é uma doença endócrina severa e é definido como uma exposição crônica a níveis elevados de glicocorticóides e, atualmente, é considerada a endocrinopatia de maior ocorrência nas clínicas veterinárias, acometendo em sua maioria, a espécie canina (Feldman et al. 2015).

A possibilidade de acometimento pelo HAC é investigada, inicialmente, com base no histórico e no exame físico, onde o animal deve apresentar um ou mais dos sintomas comuns da doença. Os testes usados com maior frequência para diagnosticar o HAC em pequenos animais são as determinações de cortisol sérico por meio do teste de supressão com baixa dose de dexametasona, relação cortisol/ creatinina urinário e estimulação com ACTH (Behrend et al. 2013).

O teste de estimulação com ACTH é utilizado não apenas como ferramenta diagnóstica,mas também como uma forma de controle desta endocrinopatia. As vantagens deste teste estendem-se pela conveniência - por ser rápido e de simples realização (Peterson 2007).

0 método de estimulação da função adrenal com ACTH resume-se na determinação do cortisol sérico antes e uma ou duas horas após a estimulação com ACTH sintéti- co (Peterson 2007). Para este, é utilizada a forma ativa da cortrosina, o tetracosactideo. 0 cortisol endógeno canino possui 39 aminoácidos, enquanto o tetracosactideo possui apenas os 24 primeiros aminoacidos desta cadeia (Martin et al. 2007). A aplicacao deste princípio ativo pode ser realizada por via endovenosa e via intramuscular e ambas demonstraram-se eficazes para a realização do estímulo da função adrenal em animais sadios, no entanto preconiza-se a via endovenosa para evitar alteraçõescoes provenientes da absorção. Na literatura, observam-se doses entre 5ug/ $\mathrm{kg}$ a $250 \mathrm{ug} /$ animal por administração endovenosa (Cohen et al. 2012) para a realização do teste, porem não relata-se dose inferior a $5 \mathrm{ug} / \mathrm{kg}$ (Behrend et al. 2013) para este fim. Como demostrado por Martin et al. (2007), a dose de 1ugkg em animais sadios promove o máximo estimulo da adrenal, constatando que uma baixa dose de ACTH pode ser igualmente ou até mais eficaz que a utilização de uma dose muito elevada do hormônio sintético, diminuindo os custos para o proprietário e respeitando a fisiologia do animal.

Na literatura nacional e internacional consultada, não constatam-se estudos que avaliam a eficácia do uso do ACTH sintético na avaliação da função adrenal em casos de HAC canino, em doses menores que as de $5 \mathrm{ug} / \mathrm{kg}$.

\section{MATERIAL E MÉTODOS}

Animais. Foram estudados 23 pacientes da espécie canina oriundos do Hospital Veterinário da Universidade Anhembi Morumbi (HVUAM). Entre os 23 pacientes, 10 animais foram classificados como sadios (grupo sadios), 6 animais portadores de hiperadrenocorticismo e em tratamento com trilostano (grupo HAC Control) e 7 animais suspeitos de HAC endógeno, que foram seriam submetidos ao teste de estímulo da funcão adrenal com ACTH sintético para fins diagnósticos (HAC Diag).

Avaliação da função adrenal. 0 grupo de animais sadios foi submetido ao teste de estimulação com ACTH sintético somente com a dose de $1 \mathrm{ug} / \mathrm{kg}$. Os pacientes do grupo HAC Control e HAC Diag foram submetidos ao teste de estimulação com ACTH sintético na dose convencional de $5 \mathrm{ug} / \mathrm{kg}$ de peso. Em um intervalo de no mínimo 24 horas e no máximo 7 dias do primeiro teste, estes pacientes foram submetidos novamente ao teste de estimulação com ACTH, com a dose proposta de $1 \mathrm{ug} / \mathrm{kg}$. Todos os pacientes pertencentes ao grupo HAC Control eram submetidos ao tratamento com trilostano (Vetoryl, Nationwide Laboratories, Inglaterra).

Coleta de material. Foram adquiridas três ampolas de Synachten $^{\circledR}$ (Novartis Pharma S.A., França), as quais foram aliquotadas em frações de 10 ug e em frações de 50 ug, ambas armazenadas em seringas de baixo volume $(0,3 \mathrm{ml}$ e $1,0 \mathrm{ml}$, respectivamente) sob congelamento, conforme recomendado pela literatura (Frank \& Oliveir 1998). No momento do procedimento, coletou-se a amostra sanguínea basal e em seguida, o ACTH sintético era retirado do armazenamento sob congelamento, induzido ao estado líquido apenas com a temperatura ambiente e então diluído em solução fisiológica, com a diluição de 4UI:100UI, em seringas de 100UI. Após esta diluição e cálculo da dose, o composto era então administrado em bolus, via endovenosa cefálica. Uma hora após, era então coletada a amostra pós ACTH. Ambas amostras foram coletadas em tubos com gel separador com ativador de coágulo, indicado para obtenção do soro sanguíneo. Os proprietários que aderiram ao estudo foram beneficiados com um desconto de $50 \%$ nos exames bioquímicos, hematológicos e urinálise completa do HVUAM e nas determinações de cortisol com o teste padrão (5ug/ kg) no Provet. 
Dosagem de cortisol. Após a coleta das amostras, estas foram levadas ao Provet sob refrigeramento onde foram destinadas à determinação de cortisol sérico por meio do radioimunoensaio.

Análise estatística. Após a obtenção dos resultados, estes foram tabulados e submetidos á análise estatística, através dos testes de Análise de Variância - ANOVA (comparação entre valores basais e após estímulo com ACTH e comparação entre as duas doses de ACTH testadas - padrão e baixa dose). Também, os testes de Kruskal-Wallis e de Dunn foram utilizados para avaliações dos valores do $\Delta$ cortisol (delta=diferença entre cortisol após estimulo e o cortisol basal) entre os grupos.

\section{RESULTADOS}

\section{Função da adrenal}

Nos pacientes Sadios $(\mathrm{n}=10)$, observou-se os valores de cortisol basal, obtidos antes da dose de $1 \mathrm{ug} / \mathrm{kg}$, em média 2,40ug/dL, desvio-padrão 1,57ug/dL, mínimo 1,56ug/dL e máximo de 4,61ug/dL. Os resultados de cortisol após a estimulação com ACTH foram de média $11,43 \mathrm{ug} / \mathrm{dL}$, desvio-padrão de 2,46ug/dL, mínimo de 7,71ug/dL e máximo de $16,48 \mathrm{ug} / \mathrm{dL}$. Ainda na dose de $1 \mathrm{ug} / \mathrm{kg}$, os resultados obtidos para o cortisol basal plasmático no grupo HAC Control $(\mathrm{n}=6)$ demonstraram média 1,53ug/dL, desvio-padrão 0,93ug/dL mínimo de 0,29ug/dL e máximo de 3,06ug/dL. Os valores de cortisol plasmático após estimulação com ACTH do grupo HAC Control sob a dose de $1 \mathrm{ug} / \mathrm{kg}$ resultaram em média $2,67 \mathrm{ug} / \mathrm{dL}$, desvio-padrão de 1,39ug/dL, mínimo de 0,51ug/dLe máximo de 3,93ug/dL. Os resultados obtidos para o cortisol basal plasmático no grupo HAC Diag $(\mathrm{n}=7)$ sob a dose de $1 \mathrm{ug} / \mathrm{kg}$ demonstraram média 3,37ug/dL, desvio-padrão 1,57ug/dL, mínimo de 0,96ug/ dL e máximo de 5,54ug/dL. Os valores de cortisol plasmático pós estimulação com ACTH do grupo HAC Diag sob a dose de $1 \mathrm{ug} / \mathrm{kg}$ resultaram em média 16,56ug/dL, desviopadrão de 7,62ug/dL, mínimo de 10,28ug/dL e máximo de $31,55 \mathrm{ug} / \mathrm{dL}$.

Os resultados obtidos para o cortisol basal plasmático no grupo HAC Control $(\mathrm{n}=6)$ sob a dose de $5 \mathrm{ug} / \mathrm{kg}$ demonstraram média 0,89ug/dL; desvio-padrão 0,23ug/dL; mínimo $0,46 \mathrm{ug} / \mathrm{dL}$ e máximo de 1,08ug/dL. Os valores de cortisol plasmático pós-estimulação com ACTH do grupo HAC Control sob a dose de $5 \mathrm{ug} / \mathrm{kg}$ resultaram em média $3,71 \mathrm{ug} / \mathrm{dL}$, desvio-padrão de 1,57ug/dL, mínimo de 1,78ug/dLe máximo de 6,02ug/dL. Os valores obtidos para o cortisol basal plasmático no grupo HAC Diag $(\mathrm{n}=7)$ sob a dose de $5 \mathrm{ug} / \mathrm{kg}$ demonstraram média 3,08ug/dL; desvio-padrão 1,99ug/ dL; mínimo 1,61 e máximo 6,72ug/dL. Os valores de cortisol plasmático pós-estimulação com ACTH do grupo HAC Diag sob a dose de $5 \mathrm{ug} / \mathrm{kg}$ resultaram em média 22,52ug/ $\mathrm{dL}$, desvio-padrão de $8,75 \mathrm{ug} / \mathrm{dL}$, mínimo de 10,84ug/dL e máximo de 30,18ug/dL. Através da análise de variância com medidas repetidas, observa-se que os grupos HAC Diag e HAC Control apresentam diferenças significativas no comportamento ao longo das avaliações $(p=0,002)$, conforme demonstrado no Quadro 1 e na Figura 1.

No grupo HAC Control, não se observa alterações significativas ao longo das avaliações $(p=0,108)$. No grupo HAC Diag observa-se alteração significativa ao longo das avaliações $(p=0,012)$. Demonstra-se acréscimo significativo en- tre o momento basal e pós ACTH com a dose de $5 \mathrm{ug} / \mathrm{kg}$ (p< 0,001 ) e entre o momento basal e pós ACTH com a dose de $1 \mathrm{ug} / \mathrm{kg}(\mathrm{p}=0,005)$. E, finalmente, não se observa diferença significativa entre as doses 1 e $5 \mathrm{ug} / \mathrm{kg}$ no momento basal $(\mathrm{p}=0,617)$ e no momento após ACTH $(\mathrm{p}=0,225)$, ou seja, ambas as doses de ACTH (1 ou $5 \mathrm{ug} / \mathrm{kg}$ ) se assemelham na resposta adrenal.

Quando avaliado o comportamento dos grupos para a dose de $1 \mathrm{ug} / \mathrm{kg}$ por meio da análise de variância com medidas repetidas, observa-se que os grupos apresentam diferenças significativas no comportamento ao longo das avaliações $(\mathrm{p}=0,001)$. Há acréscimo significativo em todos os grupos do momento basal para o momento pós ACTH: grupo HAC Control $(p=0,043)$; grupo HAC Diag $(p=0,005)$ e grupo de animais sadios $(\mathrm{p}<0,001)$, conforme demonstrado no Quadro 2, Figura 2.

\section{Delta Cortisol}

0 delta $(\Delta)$ de variação, gerado através da diferença entre os valores de cortisol após o estímulo com ACTH e o cortisol basal, demonstrou os valores, para o Grupo HAC Control $(\mathrm{n}=6)$ de média 1,14, desvio-padrão 1,04 . mediana de 0,75, mínimo de 0,22 e máximo de 2,85.Para o Grupo HAC Diag (n=7), valores de média 13,2, desvio-padrão 8,11, mediana de 10,24, mínimo de 4,74 e máximo de 28,64. Em animais Sadios ( $\mathrm{n}=10)$, observa-se média 9,03, desvio-pa-

Quadro 1. Valores de cortisol sérico (ug/dL) no teste de estimulação com ACTH em cães com hiperadrenocorticismo e em tratamento (HAC Control) e cães suspeitos de

hiperadrenocoticismo (HAC Diag). Amostras basais e pós ACTH, sob a dose de $1 \mathrm{ug} / \mathrm{kg}$ e $5 \mathrm{ug} / \mathrm{kg}$, respectivamente

\begin{tabular}{cccccccc}
\hline Grupo & Dose & Momento & $\mathrm{n}$ & Média & $\mathrm{dp}$ & Mínimo & Máximo \\
\hline HAC & $5 \mathrm{ug} / \mathrm{kg}$ & Basal & 6 & 0,89 & 0,23 & 0,46 & 1,08 \\
Control & & Pos ACTH & 6 & 3,71 & 1,57 & 1,78 & 6,02 \\
& $1 \mathrm{ug} / \mathrm{kg}$ & Basal & 6 & 1,53 & 0,93 & 0,29 & 3,06 \\
& & Pos ACTH & 6 & 2,67 & 1,39 & 0,51 & 3,93 \\
$\mathrm{HAC}$ & $5 \mathrm{ug} / \mathrm{kg}$ & Basal & 7 & 3,08 & 1,99 & 1,61 & 6,72 \\
Diag & & Pos ACTH & 7 & 22,52 & 8,75 & 10,84 & 30,18 \\
& \multirow{2}{*}{$1 \mathrm{ug} / \mathrm{kg}$} & Basal & 7 & 3,37 & 1,57 & 0,96 & 5,54 \\
& & Pos ACTH & 7 & 16,56 & 7,62 & 10,28 & 31,55
\end{tabular}

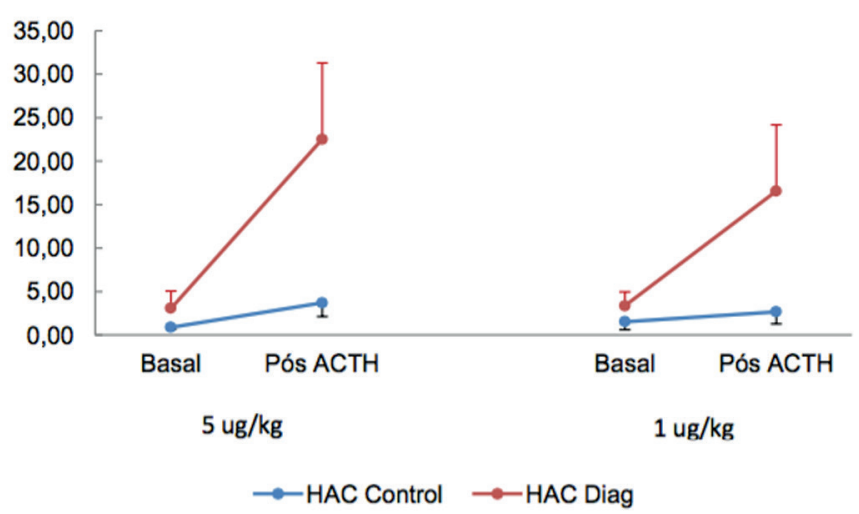

Fig.1. Representação gráfica da média dos valores de cortisol (ug/ dL) em em cães com hipera.drenocorticismo e em tratamento (HAC Control) e em cães suspeitos de hiperadrenocorticismo (HAC Diag) quando submetidos ao teste de estimulação com ACTH, sob as doses de $5 \mathrm{ug} / \mathrm{kg}$ e $1 \mathrm{ug} / \mathrm{kg}$. Valores basais e após ACTH. 
Quadro 2. Valores de cortisol sérico (ug/dL) no teste de estimulação com ACTH sob a dose de $1 \mathrm{ug} / \mathrm{kg}$ em cães com hiperadrenocorticismo e em tratamento (HAC Control), cães suspeitos de hiperadrenocorticismo (HAC Diag) e em cães sadios (Sadios)

\begin{tabular}{lcccccc}
\hline Grupo & Momento & $\mathrm{n}$ & Média & $\mathrm{dp}$ & Mínimo & Máximo \\
\hline \multirow{2}{*}{ HAC Control } & Basal & 6 & 1,53 & 0,93 & 0,29 & 3,06 \\
& Pos ACTH & 6 & 2,67 & 1,39 & 0,51 & 3,93 \\
HAC Diag & Basal & 7 & 3,37 & 1,57 & 0,96 & 5,54 \\
\multirow{4}{*}{ Sadios } & Pos ACTH & 7 & 16,56 & 7,62 & 10,28 & 31,55 \\
& Basal & 10 & 2,40 & 0,85 & 1,56 & 4,61 \\
& Pos ACTH & 10 & 11,43 & 2,46 & 7,71 & 16,48
\end{tabular}

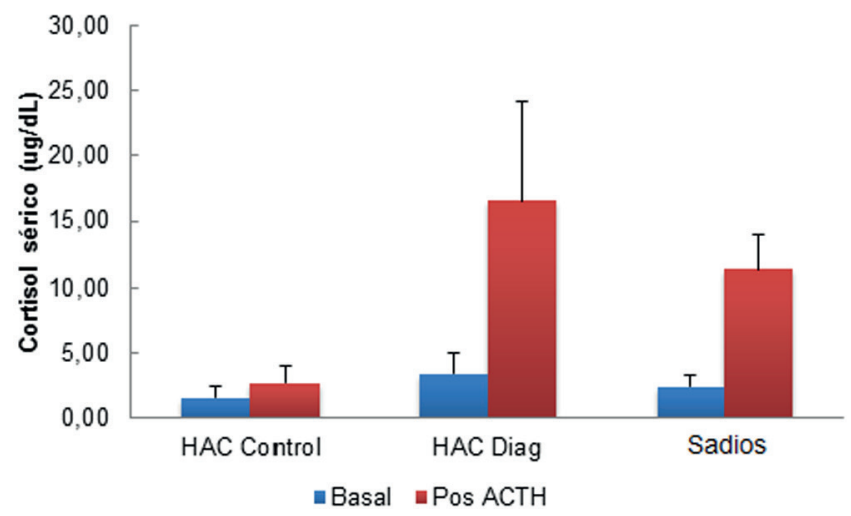

Fig.2. Representação gráfica dos valores de cortisol (ug/dL) no teste de estimulação com ACTH sob a dose de $1 \mathrm{ug} / \mathrm{kg}$, em cães sadios (Sadios) em cães com hiperadrenocorticismo e em tratamento (HAC Control) e em cães suspeitos de hiperadrenocorticismo (HAC Diag).

Quadro 3. Valores de $\Delta$-cortisol (delta = diferença entre cortisol após estimulo e o cortisol basal)em cães com hiperadrenocorticismo e em tratamento (HAC Control), em cães suspeitos de hiperadrenocorticismo (HAC Diag) e em cães sadios (Sadios)

\begin{tabular}{lccccccc}
\hline Grupo & $\mathrm{n}$ & Média & $\mathrm{dp}$ & Mediana & Mínimo & Máximo & $\mathrm{p}$ \\
\hline HAC Control & 6 & 1,14 & 1,04 & 0,75 & 0,22 & 2,85 & \\
HAC Diag & 7 & 13,2 & 8,11 & 10,24 & 4,74 & 28,64 & 0,001 \\
Sadios & 10 & 9,03 & 2,21 & 8,65 & 6,15 & 14,03 &
\end{tabular}

drão 2,21, mediana de 8,65, mínimo de 6,15 e máximo de 14,03 .

Observamos pelo teste não-paramétrico de Kruskal-Wallis que os grupos apresentam diferença significativa no delta de variação do momento basal para o momento pós ACTH $(\mathrm{p}=0,001)$, conforme demonstrado no Quadro 3 e na Figura 3. Pelo teste de Dunn observamos que o grupo Controle apresenta delta significativamente menor que o dos grupos diagnóstico $(\mathrm{p}<0,05)$ e animais sadios $(\mathrm{p}<0,05)$.

As demais comparações não apresentaram diferença significativa.

\section{DISCUSSÃO}

Os testes diagnósticos disponíveis para avaliar o possível acometimento por hiperadrenocorticismo em cães possuem certa diversidade. Entre os mais utilizados, estão o teste de estimulação com ACTH e o teste de supressão com baixa dose de dexametasona. No entanto, o teste de estimulação

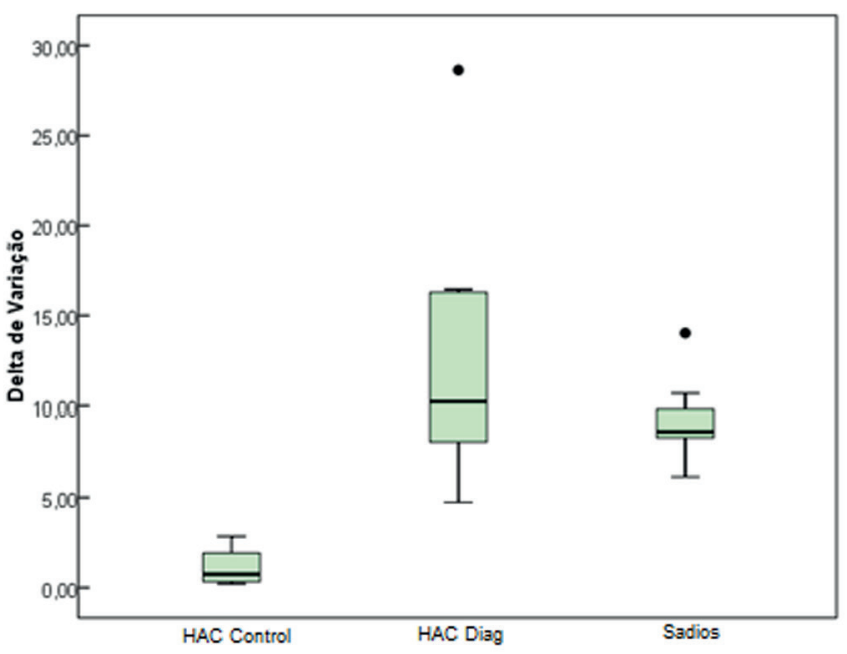

Fig.3. Representação gráfica dos valores de $\Delta$-cortisol (delta = diferença entre cortisol após estimulo e o cortisol basal) dos em cães sadios (Sadios) em cães com hiperadrenocorticismo e em tratamento (HAC Control) e em cães suspeitos de hiperadrenocorticismo (HAC Diag).

com ACTH é conhecido por sua conveniência e rapidez (especialmente quando comparado ao teste de supressão com baixa dose de dexametasona), sendo pré-requisito para o acompanhamento da doença em animais em tratamento (Behrend et al. 2013).

0 hiperadrenocorticismo é um distúrbio de alta incidência em cães, sendo bem mais comum do que em outras espécies, como em felinos e em humanos (Rijnberk et al. 2003). 0 hiperadrenocorticismo canino é diagnosticado com base na história clínica, sinais e sintomas, exames complementares de triagem e, indipensavelmente, os testes específicos para diagnóstico (Behrend et al. 2013). Os testes de rotina, no entanto, revelam alterações que auxiliam apenas na suspeita não específica do hiperadrenocorticismo (Reusch et al. 1991). A estimulação com ACTH pode ser utilizada tanto para diagnóstico como para acompanhamento de animais já tratados (Peterson 2007), sendo considerado o teste de eleição para o controle da doença (Feldman et al. 2015). A principal indicação para a busca do diagnóstico da síndrome é a presença de um ou mais sinais clínicos da doença, no entanto quanto mais anormalidades forem identificadas, maior será a indicação para a realização dos testes diagnósticos específicos (Behrend et al. 2013). 0 teste de estimulação com ACTH é utilizado para avaliar tanto a hiperfunção quanto hipofunção adrenal. Considera-se o teste eficaz para identificar o hiperadrenocorticismo canino quando este é capaz de induzir a resposta exacerbada da função adrenal através da administração de uma dose recomendada de ACTH sintético.

0 teste de estímulo da função adrenal, além de ser utilizado para o diagnóstico e acompanhamento do hiperadrenocorticismo; também é utilizado para identificar pacientes com supressão parcial da adrenal através do tratamento com esteróides, para avaliar o risco de desenvolvimento de complicações advindas da retirada abrupta da medicação (Frank et al. 2000).

A dose atualmente recomendada para realizar o teste de estimulação com ACTH em cães é de $5 u g / k g$. Conforme 
demonstrado por Frank et al. (2000), a dose de 5ug/kg do tetracosactídeo provoca uma maior resposta do que a dose de $250 \mathrm{ug} /$ animal após 60 minutos, incitando a especulação de que menores doses de ACTH podem ser eficazes para realizar o estímulo da função adrenal adequadamente.

0 presente trabalho objetivou avaliar a eficácia de uma menor dose de ACTH sintético $(1 \mathrm{ug} / \mathrm{kg})$ quando aplicado o teste de estimulação com ACTH em três distintas modalidades - animais sadios, animais suspeitos (HAC Diag) ou avaliação de tratamento dos pacientes já portadores (HAC Control). Esta avaliação foi baseada na mensuração do cortisol basal sérico e no cortisol sérico após estimulação com ACTH deste três grupos.

No grupo de animais sadios, como a dose padrão de $5 \mathrm{ug} / \mathrm{k}$ já é consagrada e amplamente divulgada pela literatura em seus valores de normalidade, foi testada somente a dose de 1ug/kg. Entretanto, para efeitos de comparação, nos grupos HAC Control e HAC Diag foi também testada a dose de $5 \mathrm{ug} / \mathrm{kg}$ recomendada pela literatura (Martin et al. 2007, Feldman et al. 2015 ) paralelamente à a dose proposta de $1 \mathrm{ug} / \mathrm{kg}$, observando sua adequação na atividade estimulatória da glândula adrenal.

Os pacientes testados no grupo de animais sadios apresentaram valores de cortisol pós ACTH na concentração desejada $(<17 \mathrm{ug} / \mathrm{dL})$, excluindo a presença de hiperadrenocorticismo e possíveis doenças não adrenais, sendo utilizado como base de comparação para os grupos HAC Control e HAC Diag. Doenças de caráter agudo podem ocasionar mudanças significativas na função endócrina, alterando os resultados do teste, ocasionando valores de cortisol sérico normais a aumentados, conforme demonstrado por Martin et al. (2008).

O grupo de animais suspeitos (HAC Diag) foi selecionado com base no histórico e anamnese, sinais clínicos compatíveis com a doença (Behrend et al. 2013) e, ocasionalmente, acompanhados de testes laboratoriais de triagem. Além destes cuidados, os proprietários foram questionados quanto á utilização de medicações que poderiam influenciar o funcionamento do eixo hipotalamico hipofisário adrenal. Em estudos anteriores, foi constatado que drogas como esteróides, a metoclorpramoda, a clonidina, a buprenorfina, a codeína, clomiparmina, o ceruletídeo e, especialmente, os progestágenos e o glicocorticóides, sendo estes inibidores do eixo hipotalämico hipofisário adrenal (Behrend et al. 2013).

Para os animais com suspeita clínica (HAC Diag), os valores encontrados de cortisol sérico após ACTH sob a dose de $5 \mathrm{ug} / \mathrm{kg}$ resultaram em média 22,52ug/dL, mínimo de $10,84 \mathrm{ug} / \mathrm{dL}$ e máximo de $30,18 \mathrm{ug} / \mathrm{dL}$, ao passo que sob a dose de $1 \mathrm{ug} / \mathrm{kg}$, observaram-se os resultados de média 16,56ug/dL, mínimo de 10,28ug/dL e máximo de 31,55ug/ dL. Confirma-se o acometimento do paciente pela síndrome quando estes valores estão acima de 20ug/dL quando realizado o teste sob a dose de $5 \mathrm{ug} / \mathrm{kg}$ (Peterson 2007), observando-se uma resposta exagerada ao estímulo com a cortrosina em 4 entre 7 animais testados com esta dose produziram valores superiores a $20 \mathrm{ug} / \mathrm{dL}$, enquanto na dose de $1 \mathrm{ug} / \mathrm{kg}, 2$ animais mostraram-se portadores. São considerados animais suspeitos aqueles que apresentam valores entre $18 \mathrm{ug} / \mathrm{dL}$ e $20 \mathrm{ug} / \mathrm{dL}$ de cortisol sérico após o estímulo. No grupo de animais suspeitos (HAC Diag), com a dose convencional de $5 \mathrm{ug} / \mathrm{k}$, nenhum animal entre 7 animais apresentou este valor limítrofe, no entanto, na dose de $1 \mathrm{ug} / \mathrm{kg}$, apenas um animal apresentou valor suspeito (18 ug/Dl) entre os 7 animais. 0 número de animais do grupo HAC Diag que apresentaram valores de cortisol pós ACTH inferiores aos valores considerados para animais suspeitos (<18ug/Dl) foram de 3/7 animais na dose de 5ug/kg e 4/7 animais na dose de $1 \mathrm{ug} / \mathrm{kg}$. Estas concentrações oriundas da hiperfunção adrenal não são capazes de diferenciar o hiperadrenocorticismo hipófise dependente e o adrenal dependente (Cook \& Bonda 2010). No entanto, conforme demonstrado por Reusch et al. (1991), quando realizado o teste de estimulação com ACTH em pacientes com carcinomas adenocorticais, a concentração de cortisol sérico foi significativamente maior do que no grupo portador de adenomas adrenocorticais em 41 animais testados.

Sabe-se que mesmo que um animal apresente os sinais clínicos da doença, considera-se que este está em fase de desenvolvimento do quadro ou pode estar acometido com o hiperadrenocorticismo atípico (oculto); modalidade da síndrome cujos testes diagnósticos específicos não demonstram-se eficientes; tornando necessário a dosagem de hormonios sexuais que pode ser realizada através de um painel andrológico ou de dosagens específicas para hormonios como a 17-hidroxiprogesterona (Behrend et al. 2013).

Uma vez que o diagnóstico do hiperadrenocortismo canino é realizado, é necessário analisar se há a possibilidade de tratamento e qual será a medicação utilizada. Alguns proprietários, no entanto, hesitam em tratar seus animais mesmo com a confirmação diagnóstica, em função do nível de comprometimento e dos riscos associados (Reine et al. 2007).

Entre os fármacos de eleição para o tratamento do hiperadrenocorticismo canino, são os mais utilizados o mitotano e o trilostano (Reine et al. 2007); além de tratamentos alernativos como o L-Deprenyl, a bromocriptina, o ciproeptadíneo, e a combinação de metyrapone e aminogluthimide (Feldman et al. 2015). 0 mitotano e o trilostano são considerados fármacos eficazes para tratamento de cães com hiperadrenocorticismo e, mesmo que existam diferenças em seus protocolos de aplicação, efeitos colaterais e monitoração, ambos os fármacos podem ser utilizados para este fim (Reine et al. 2007).

Todos os animais que foram submetidos ao teste para controle da doença eram medicados com trilostano. 0 trilostano é uma medicação inibidora da síntese do cortisol, de forma indireta, através de bloqueio enzimático da enzima 3-beta hidroxiesteróide desidrogenase e sua ação demonstra grande eficácia, sendo assim possível monitorar os resultados terapeuticos objetivamente ao utilizar o teste de estimulação com ACTH (Feldman et al. 2015). Em animais com hiperadrenocorticismo hipófise dependente 0 trilostano mosta-se eficaz ao reduzir significativamente o cortisol sérico (Sieber-Ruckstuhl et al. 2005). Sabe-se que a terapia com o trilostano não apenas altera as concentracões de cortisol, mas também minimamente as concentrações de aldosterona e, conforme demonstrado por Sieber- 
-Ruckstuhl et al. (2005), a medicação também ocasiona aumento significativo da 17-alfa-hidroxiprogesterona e da desidroepiandrosterona devido ao bloqueio enzimático. No entanto, o trilostano é considerado uma forma eficaz de tratamento do hiperadrenocorticismo atípico pois este diminui significativamente os níveis de cortisol, fenômeno provavelmente ocasionado por seu efeitos mais distais no metabolismo hormonal (Sieber-Ruckstul et al. 2005). De acordo com a literatura, indica-se monitoração mensal de pacientes tratados com trilostano, através do teste de estimulação com ACTH. Após avaliação mensal realizada por três meses, indica-se um acompanhamento a cada três meses no primeiro ano e de quatro a seis meses após um ano (Ramsey et al. 2010), demonstrando a necessidade da realização do exame em animais em tratamento.

Animais tratados com trilostano devem receber a medicação com a dose prescrita de 2 a 4 horas antes do exame, para que no momento do teste a medicação esteja exercendo seu maior potencial inibitório, conforme demonstrado por Bonadio et al. (2014). Este protocolo foi aplicado aos animais do grupo HAC Control (animais portadores em tratamento) no presente estudo.

Os grupos HAC Diag e HAC Control foram submetidos ao teste de estimulação com ACTH com as doses de $5 \mathrm{ug} / \mathrm{kg}$ e $1 \mathrm{ug} / \mathrm{kg}$, respectivamente; com o período de uma semana entre o primeiro e o segundo. De acordo com a literatura, após a injeção do ACTH sintético (em bolus) o composto permanece no organismo do animal por aproximadamente 120 minutos e, após este período, as concentrações de cortisol endógeno voltam á sua normalidade (Greco et al. 1998). No entanto, foi escolhido o intervalo de uma semana entre as aplicações para evitar uma possível estimulação oriunda de efeito residual do composto (Neiger et al. 2002) e evitar também mudanças na rotina e no estado de saúde do animal que pudessem alterar as concentrações de cortisol sérico (Church et al. 1994), atentando a sensibilidade do teste. Fatores de estresse como hospitalização, manipulação e outros fatores podem resultar em elevações no cortisol basal de animais sadios (Cayzer \& Jones 1993).

Entre os grupos observou-se que houve diferença significativa nos níveis de cortisol sérico perante o estímulo com o ACTH. No entanto observou-se que o delta cortisol do grupo HAC Control demonstrou-se inferior ao dos outros grupos, como mostrado pelo teste de Dunn. Isto se dá pelo efeito terapêutico desejado do uso do trilostano, qual seja o de diminuir as concentrações de cortisol sérico - basal e pós ACTH. Considera-se eficaz o tratamento para o hiperadrenocorticismo quando as concentrações de cortisol sérico após estimulação com ACTH alcançam um mínimo de $1 \mathrm{ug} / \mathrm{dL}$ e máximo de $5 \mathrm{ug} / \mathrm{dL}$, no entanto, resultados com até $9 \mathrm{ug} / \mathrm{dL}$ no pós ACTH podem ser aceitas caso o paciente esteja sob bom controle clínico (Feldman et al. 2015). Os valores de cortisol plasmático após estimulação com ACTH do grupo HAC Control sob a dose de 1ug/ kg resultaram em média 2,67ug/dL, mínimo de $0,51 \mathrm{ug} / \mathrm{dL}$ e máximo de 3,93ug/dL. Os valores de cortisol plasmático pós-estimulação com ACTH do grupo HAC Control sob a dose de $5 \mathrm{ug} / \mathrm{kg}$ resultaram em média 3,71ug/dL, mínimo de 1,78ug/dLe máximo de 6,02ug/dL. Na dose de 5ug/kg,
2 entre 7 animais demonstraram valores de cortisol basal e pós-ACTH conforme o desejado ( $>1 \mathrm{ug} / \mathrm{dL}$ e $<5 \mathrm{ug} / \mathrm{dL}$, respectivamente) e com a dose de $1 \mathrm{ug} / \mathrm{kg}, 3$ entre 7 animais apresentaram estes valores. Entre os 7 animais estudados neste grupo, na dose de $5 \mathrm{ug} / \mathrm{dL}, 4$ animais demonstraram valores basais inferiores a $1 \mathrm{ug} / \mathrm{dL}$ e na dose de $1 \mathrm{ug} / \mathrm{kg}, 3$ animais. Não foram encontrados valores superiores a 9ug/ dL no momento pós-ACTH nos grupo analisados através do estímulo com as diferentes doses.

A intenção em utilizar uma menor dose para a realização do teste de estimulação com ACTH baseia-se no fato de que a ocupação de apenas uma pequena fração dos receptores para ACTH pode resultar no total estímulo da esteroidogenese (Frank et al. 2000), portanto uma baixa dose ocasionaria ação suficiente para avaliar a função adrenal de forma mais próxima à fisiologia do animal (Martin et al. 2007), conforme demonstrado no presente estudo; para animais sadios, suspeitos e portadores em tratamento da doença.

Os resultados apresentados neste trabalho sugerem a eficácia do teste sob esta dose menor, ao comparar o cortisol sérico após a estimulação com a dose menor de ACTH com a dose convencional. Para maior demonstração desta eficácia, estudos complementares, com um número maior de animais, devem ser desenvolvidos.

\section{CONCLUSÕES}

Com base nos dados obtidos com o presente trabalho conclui-se que pode ser adequado realizar o teste de estímulo com ACTH com uma menor dose pois, conforme observado nos resultados analisados, esta dose promove a resposta adrenal semelhante que a dose proposta pela literatura.

A utilização de uma menor dose de ACTH no teste de estímulo da função adrenal representa não apenas uma avaliação do eixo adequada, como também uma redução significativa no custo do teste, possibilitando a maior adesão do proprietário ao procedimento.

Sabe-se que este teste é o teste de escolha para avaliação e acompanhamento de animais portadores de HAC tratados com mitotano ou trilostano, logo propões-se uma possível redução de custo neste procedimento indispensável para acompanhamento do paciente.

Concluiu-se, também, que não apenas o ACTH sintético pode ser armazenado sob congelamento em seringas de baixo volume, mas também pode ser congelado sob diluição com solução fisiológica, preservando sua eficácia.

\section{REFERÊNCIAS}

Behrend E., Kooistra H., Nelson R., Reusch C. \& Scott-Moncrieff J. 2013. Diagnosis of spontaneous canine Hyperadrenocorticism: 2012 ACVIM consensus statement (small animal). J. Vet. Intern. Med. 27(6):12921304.

Bonadio C., Feldman E., Cohen T.E. \& Kass P. 2014. Comparison of adrenocorticotropic hormone stimulation test results started 2 versus 4 hours after trilostane administration in dogs with naturally occurring hyperadrenocorticism. J. Vet. Intern. Med. 28(4):1239-1243.

Cayzer J. \& Jones B. 1993. Canine hyperadrenocorticism. N.Z. Vet. J. 41(2): 53-68.

Church D.B., Nicholson A.I., Ilkiw J.E. \& Emslie D.R. 1994. Effect of non-ad- 
renal illness, anaesthesia and surgery on plasma cortisol concentrations in dogs. Res. Vet. Sci. 56(1):129-131.

Cohen T. \& Feldman E. 2012. Comparison of IV and IM formulations of synthetic ACTH for ACTH stimulation tests in healthy dogs. J. Vet. Intern. Med. 26(2):412-414.

Cook A. \& Bonda K. 2010. Evaluation of the use of baseline cortisol concentration as a monitoring tool for dogs receiving trilostane as a treatment for hyperadrenocorticism. J. Am. Vet. Med. Assoc. 237(7):801-805.

Feldman E.C., Nelson R.W., Reusch C., Scott-Moncrieff J.C.R. \& Behrend E.N. 2015. Canine and Feline Endocrinology. W.B Saunders, St Louis, MO. 669p.

Frank L.A. \& Oliver J. 1998. Comparison of serum cortisol concentrations in clinically normal dogs after administration of freshly reconstituted versus reconstituted and stored frozen cosyntropin. J. Am. Vet. Med. Assoc. 212:1569-1571.

Frank L.A., Denovo R.C., Kraje A.C. \& Oliver J.W. 2000. Cortisol concentrations following stimulation of healthy and adrenopathic dogs with two doses of tetracosactrin. J. Small Anim. Pract. 41(7):308-311.

Greco D., Behrend E., Brown S., Rosychuck R. \& Groman R. 1998. Pharmacokinetics of exogenous corticotropin in normal dogs, hospitalized dogs with non adrenal illness and adrenopathic dogs. J. Vet. Pharmacol. Therapeut. 21(5):369-374.

Martin L.G., Groman R.P., Fletcher D.J., Behrend E.N., Kemppainen R.J., Moser V.R. \& Hickey K.C. 2008. Pituitary-adrenal function in dogs with acute critical illness. J. Am. Vet. Med. Assoc. 233(1):87-95.
Martin L.G., Behrend E.N., Mealey K.L., Carpenter D.M. \& Hickey K.C. 2007. Effect of low doses of cosyntropin on serum cortisol concentrations in clinically normal dogs. J. Am. Vet. Med. Assoc. 230(9):1339-1339.

Neiger R., Ramsey I., O’Connor J., Hurley K.J. \& Mooney C.T. 2002. Trilostane treatment of 78 dogs with pituitary-dependent hyperadrenocorticism. Vet. Rec. 150(26):799-804.

Peterson M. 2007. Diagnosis of hyperadrenocorticism in dogs. Clin. Techn. Small Anim. Pract. 22(1):2-11.

Ramsey I.K. 2010. Trilostane in dogs. Vet. Clin. North Am., Small Anim. Pract. 40(2):269-283.

Reine J.N. 2007. Medical management of pituitary-dependent hyperadrenocorticism: mitotane versus trilostane. Clin. Techn. Small Anim. Pract. 22(1):18-25.

Reusch C. \& Feldman E. 1991. Canine hyperadrenocorticism due to adrenocortical neoplasia: pretreatment evaluation of 41 dogs. J. Vet. Intern. Med. 5(1):3-10.

Rijnberk A., Kooistra H.S. \& Mol J. 2003. Endocrine diseases in dogs and cats: similarities and differences with endocrine diseases in humans. Growth Hormone IGF Res. 13:S158-S164.

Sieber-Ruckstuhl N., Boretti F., Wenger M., Maser-Gluth C. \& Reusch C. 2006. Cortisol, aldosterone, cortisol precursor, androgen and endogenous ACTH concentrations in dogs with pituitary-dependant hyperadrenocorticism treated with trilostane. Domest. Anim. Endocrinol. 31(1):63-75. 